\title{
Inhibition of Azoxymethane-induced Colorectal Aberrant Crypt Foci in Mice Fed a High-fat Diet by Pleurotus eryngii (Eringi) and Hypsizygus marmoreus (Bunashimeji)
}

\author{
Takaaki Kaneko', Takeshi Chihara ${ }^{1 *}$, Kan Shimpo ${ }^{1}$, Hidehiko Beppu ${ }^{1}$, Takashi \\ Higashiguchi $^{2}$, Shigeru Sonoda ${ }^{1}$
}

\begin{abstract}
Obesity markedly increases the risk of colorectal cancer. Recently, the preventive effects of edible mushrooms on triglyceride elevation and visceral fat accumulation have been reported. Here, the effects of Pleurotus eryngii (Eringi) and Hypsizygus marmoreus (Bunashimeji) on azoxymethane (AOM)-induced aberrant crypt foci (ACF; precancerous lesions) in the colorectums of mice fed a high-fat diet were examined. Eringi (ER) and Bunashimeji (BU) mushroom powder samples were used. Six-week-old male C57BL/6J mice received an intraperitoneal injection of AOM $(10 \mathrm{mg} / \mathrm{kg})$ once a week for two weeks, and were sacrificed and dissected at 6 weeks after the start of the experiment. After the initiation of the experiment, they received a normal diet (ND), high-fat diet (HFD), HFD + ER (1 or 5\% of diet), or HFD + BU ( 1 or $5 \%$ of diet). As a result, body and fat weights were significantly lower in the 5\% ER and BU groups than in the HFD group. Liver triglyceride levels were also significantly lower in the 5\% ER and BU groups. Total liver cholesterol levels were significantly lower in the 5\% ER group. The numbers of ACF (especially large ACF) showed strong inhibitory effects in both ER and BU groups. Measurement of the cell proliferation marker Ki-67 labeling index in the colonic mucosa demonstrated more significant suppression in both ER and BU groups than in the HFD group. These results suggest that the simultaneous intake of ER and BU may inhibit colorectal tumorigenesis in HFD-fed mice.
\end{abstract}

Keywords: Pleurotus eryngii (Eringi) - Hypsizygus marmoreus (Bunashimeji) - aberrant crypt foci (ACF)

Asian Pac J Cancer Prev, 16 (9), 3881-3885

\section{Introduction}

In Japan, the age-adjusted mortality due to colon cancer increased in both men and women from the postwar era to the mid-1990s, and, subsequently, gradually decreased (Cancer Information Service, 2015). Site-specific cancer mortality rates were: lung cancer, $23.9 \%$, gastric cancer, $15.0 \%$, and colon cancer, $11.9 \%$ (third-leading cause of death among men) in men, and colon cancer, $14.9 \%$ (firstleading cause of death among women) in women (Ministry of Health, Labour and Welfare, 2012).

Dietary habits, such as excessive calorie and animal fat intake, particularly meat, alcohol, and fat ingestion, are associated with the development of colon cancer. Excessive fat intake, as well as obesity, increases the risks of hyperlipidemia, arteriosclerosis (Miyazaki et al., 2010), and colon carcinogenesis (Bardou et al., 2013). As recently reported, visceral fat obesity is strongly correlated with the risk of colon carcinogenesis (Endo et al., 2013).

Mushrooms have physiological activities, such as antitumor activity (Ren et al., 2012), cardiovascular disease-preventing effects (Guillamon et al., 2010), hypoglycemic activity (Jayasuriya et al., 2012), and cholesterol-lowering effects (Jeong et al., 2010; Sato et al., 2013). Ohtsuki et al. (2007) reported that Bunashimeji had inhibitory effects on hepatic triglyceride accumulation and cholesterol-lowering effects.

Mizutani et al. (2008) reported that an Eringi extract inhibited pancreatic lipase activity in a dosedependent manner in an in vitro study and suppressed serum triglyceride elevation in mice and humans after fat loading. Mori et al. (2008) reported that Eringi, Maitake, and Bunashimeji, particularly Bunashimeji, had antiatherosclerotic effects in mice fed a $3 \%$ freeze-dried powder diet. Bunashimeji also exhibited antioxidant effects (Matsuzawa et al., 1997; 1998) and antitumor activity (Ikekawa et al., 1992).

Eringi may prevent visceral fat accumulation and suppress colon carcinogenesis in mice by strongly inhibiting pancreatic lipase activity and suppressing triglyceride elevation. Bunashimeji may suppress colon carcinogenesis through antioxidant effects, antitumor activity, and preventive effects on visceral fat accumulation. 
In the present study, the effects of Eringi and Bunashimeji on colorectal precancerous lesions (aberrant crypt foci, $\mathrm{ACF}$ ) were examined in azoxymethane (AOM)induced mice under high-fat dietary conditions.

\section{Materials and Methods}

This experiment was conducted in accordance with Endo et al. (2009).

\section{Animals and diet}

Five-week-old male C57Bl/6J mice were purchased from Japan SLC Inc. (Hamamatsu, Japan). They were kept in groups of two or three in plastic cages with woodchip bedding and fed a normal diet (ND; AIN-93G, Oriental East Co. Ltd., Tokyo, Japan) in an animal facility controlled at a temperature of $23 \pm 5^{\circ} \mathrm{C}, 60 \pm 5 \%$ humidity, and with 12-h light/dark cycle. The care and use of the animals were in accordance with the 'Guidelines for the Management of Laboratory Animals at Fujita Health University' and the experimental protocols were approved by the Institutional Animal Care and Use Committee of Fujita Health University.

Eringi (ER) and Bunashimeji (BU) mushroom powder samples were provided by Hikuto Corporation (Nagano, Japan) and added to a high fat diet (HFD-60, Oriental East Co. Ltd., Tokyo, Japan) at 5\% and 1\%, respectively.

\section{Experimental protocol}

After acclimation for 1 week, mice were divided into 6 groups. Group 1 (ND, n=10) was given ND, Group 2 (HFD, $\mathrm{n}=10$ ) was given HFD, Group 3 (HFD+5\%ER, $\mathrm{n}=10)$ was given HFD containing $5 \%$ ER, Group 4 (HFD+1\%ER, $\mathrm{n}=10$ ) was given HFD containing $1 \% \mathrm{ER}$, Group 5 (HFD+5\%BU, $\mathrm{n}=10$ ) was given HFD containing $5 \% \mathrm{BU}$, and Group 6 (HFD+1\%BU, $\mathrm{n}=10)$ was given HFD containing $1 \%$ BU. All mice were fed for 6 weeks, with tap water ad libitum. After one week, all mice were given intraperitoneal injections of $10 \mathrm{mg} / \mathrm{kg}$ body weight AOMonce a week for two weeks. At 6 weeks after the start of the experiment, all mice were anesthetized with Nembutal, exsanguinated via the heart into heparincoated syringes, and carefully autopsied. The colorectum, principal organs (liver, kidneys, spleen) and fat tissues (epididymis, posterior abdominal wall, mesentery) were immediately removed. The weights of principal organs and fat tissues were measured. The livers were stored at $-80^{\circ} \mathrm{C}$ until determination of triglyceride and total cholesterol concentrations.

\section{Determination of liver triglyceride and total cholesterol concentrations}

According to the method of Edvardsson et al. (2006), frozen livers were homogenized in isopropanol $(50 \mathrm{mg}$ tissue $/ 1 \mathrm{ml}$ ) and incubated at $4^{\circ} \mathrm{C}$ for $1 \mathrm{~h}$. The samples were centrifuged at $4^{\circ} \mathrm{C}$ for $5 \mathrm{~min}$ at $500 \times \mathrm{g}$, and triglyceride and total cholesterol concentrations in the supernatants were measured with Triglyceride E-Test Wako and Cholesterol E-Test Wako kits (Wako Pure Chemical Industries, Ltd., Osaka, Japan) according to the manufacturer's protocol. Analysis of aberrant crypt foci
The removed colorectum was gently flushed with saline to remove any fecal contents, opened longitudinally, and fixed in $10 \%$ neutralized formalin. After fixation, each colon was rinsed in PBS, stained with $0.2 \%$ methylene blue, and microscopically observed. Identification of ACF was carried out according to the criteria described previously (McLellan et al.,1998), and the amount of ACF formation was determined.

\section{Immunohistochemistry}

After analysis of ACF, the colorectum was processed to perform immunohistochemical detection of Ki-67, a cell proliferation marker. Ki-67 was stained to compare the positivity rate. Epithelial proliferation of the colon was determined by a slight modification of the method of Katsuki et al. (2006) and Kaneko et al. (2012). Briefly, the sections were retrieved in an autoclave in citrate buffer $(\mathrm{pH}$ 6.0 ) for $20 \mathrm{~min}$ at $120^{\circ} \mathrm{C}$. Endogenous peroxidase activity was blocked by incubation of slides in absolute methanol containing $3 \% \mathrm{H}_{2} \mathrm{O}_{2}$ for $15 \mathrm{~min}$ at room temperature. They were then incubated with anti-Ki-67 (rat anti-mouse Ki-67 antigen clone TEC-3, DakoCytomation, Denmark) at a working dilution of 1:100. After $30 \mathrm{~min}$ at room temperature, they were treated with Histofine Simple Stain Mouse MAX-PO (Rat) reagent (Nichirei Bioscience, Tokyo, Japan) for $30 \mathrm{~min}$. They were washed three times with PBS after each incubation, and 3,3'-diaminobenzidine was employed as a chromogen. The nuclei were lightly counterstained with Mayer's hematoxylin solution. For determination of immunostaining, epithelial cells of the colon were counted from the lowest point to the tip of the crypt by light microscopy using $400 \times$ magnification. The number of positively stained cells in each crypt column was recorded. The results were defined as the ratio of the number of positive-stained cells to the total number of cells counted (at least 500), and then multiplied by 100 .

\section{Data analysis}

Data are presented as the mean \pm S.E. Statistical comparisons were made with Student's $t$-test or two-way analysis of variance where appropriate. These analyses were performed using Instat version 3.0 for Windows (GraphPad Software, Inc., San Diego, CA, USA). Differences were considered to be significant at $\mathrm{p}<0.05$.

\section{Results}

\section{General observations}

Food consumption and final body and organ weights are shown in Table 1. Food consumption was not significantly different among the groups. Body weights were significantly higher in Group 2 than in Group 1 and significantly lower in Groups 3 and 5 than in Group 2 at one week after the start of the experiment. The weight change was observed throughout the experimental period. The absolute liver weight was significantly higher in Group 2 than in Group 1 ( $\mathrm{p}<0.05)$, and was significantly lower in Groups 3 and 5 than in Group $2(\mathrm{p}<0.01)$. Similarly, the absolute kidney weight was significantly higher in Group 2 than in Group $1(\mathrm{p}<0.01)$, and was significantly lower in Groups 3 and 5 than in Group 2 
Inhibition of AOM-induced Colorectal Aberrant Crypt Foci in Mice Fed a High-fat Diet by Mushrooms Table 1. Food Consumption, Final Body Weight, and Organ Weight in AOM-treated C57BL/6J Mice

\begin{tabular}{|c|c|c|c|c|c|c|}
\hline $\begin{array}{l}\text { Group } \\
\text { Diet }\end{array}$ & $\begin{array}{c}1 \\
\text { Normal diet }\end{array}$ & $\begin{array}{c}2 \\
\text { HFD }\end{array}$ & $\begin{array}{c}3 \\
\mathrm{HFD}+5 \% \mathrm{PE}\end{array}$ & $\begin{array}{c}4 \\
\mathrm{HFD}+1 \% \mathrm{PE}\end{array}$ & $\begin{array}{c}5 \\
\text { HFD+5 }\end{array}$ & $\begin{array}{c}6 \\
\mathrm{HFD}+1 \% \mathrm{HM}\end{array}$ \\
\hline Number of animals & 10 & 10 & 10 & 10 & 10 & 10 \\
\hline $\begin{array}{l}\text { Food consumption } \\
\text { (g/kg/day) }\end{array}$ & $101.0 \pm 2.0$ & $118.6 \pm 26.1$ & $94.4 \pm 8.6$ & $137.1 \pm 43.2$ & $126.6 \pm 35.2$ & $88.3 \pm 3.0$ \\
\hline Final body weight (g) & $24.3 \pm 0.8$ & $31.5 \pm 0.9+\dagger$ & $23.9 \pm 0.5 * *$ & $29.9 \pm 1.0$ & $25.2 \pm 0.5 * *$ & $29.3 \pm 0.6$ \\
\hline \multicolumn{7}{|l|}{ Absolute organ weight } \\
\hline Liver $(\mathrm{g})$ & $0.82 \pm 0.02$ & $0.99 \pm 0.04 \dagger$ & $0.77 \pm 0.02 * *$ & $0.95 \pm 0.04$ & $0.77 \pm 0.02 * *$ & $0.91 \pm 0.03$ \\
\hline Kidneys (g) & $0.25 \pm 0.01$ & $0.30 \pm 0.01 \dagger \dagger$ & $0.24 \pm 0.01 * *$ & $0.28 \pm 0.01$ & $0.27 \pm 0.01 *$ & $0.29 \pm 0.01$ \\
\hline Spleen $(\mathrm{g})$ & $0.07 \pm 0.00$ & $0.09 \pm 0.01 \dagger$ & $0.07 \pm 0.00 * *$ & $0.08 \pm 0.00$ & $0.08 \pm 0.01$ & $0.09 \pm 0.00$ \\
\hline \multicolumn{7}{|c|}{ Relative organ weight (100 g body weight) } \\
\hline Liver $(\mathrm{g})$ & $3.38 \pm 0.05$ & $3.16 \pm 0.10$ & $3.23 \pm 0.03$ & $3.17 \pm 0.05$ & $3.04 \pm 0.06$ & $3.10 \pm 0.06$ \\
\hline Kidneys (g) & $1.04 \pm 0.02$ & $0.95 \pm 0.03 \dagger$ & $0.99 \pm 0.01$ & $0.94 \pm 0.02$ & $1.08 \pm 0.02 * *$ & $1.01 \pm 0.02$ \\
\hline Spleen $(\mathrm{g})$ & $0.29 \pm 0.02$ & $0.28 \pm 0.01$ & $0.29 \pm 0.01$ & $0.27 \pm 0.00$ & $0.30 \pm 0.02$ & $0.31 \pm 0.01$ \\
\hline
\end{tabular}

Abbreviations: HFD, High-fat diet; PE, Pleurotus eryngii (Eringi); HM, Hypsizygus marmoreus (Bunashimeji); Significantly different from Group $1\left(\dagger \mathrm{p}<0.05 ; \dagger \uparrow \mathrm{p}<0.01\right.$; Student $t$-test): Significantly different from Group $2\left(* * \mathrm{p}<0.01,{ }^{*} \mathrm{p}<0.05\right.$; Dunnett's multiple comparisons test)

$(\mathrm{p}<0.01$ and $\mathrm{p}<0.05$, respectively). The absolute spleen weight was significantly higher in Group 2 than in Group $1(\mathrm{p}<0.05)$, and was significantly lower in Group 3 than in Group $2(\mathrm{p}<0.01)$. These results reflect weight gains induced by HFD diets. No significant difference was noted in relative liver and spleen weights. However, the absolute kidney weight was significantly lower in Group 2 than in Group $1(\mathrm{p}<0.05)$, and was significantly higher in Group 5 than in Group $2(\mathrm{p}<0.01)$ and comparable between
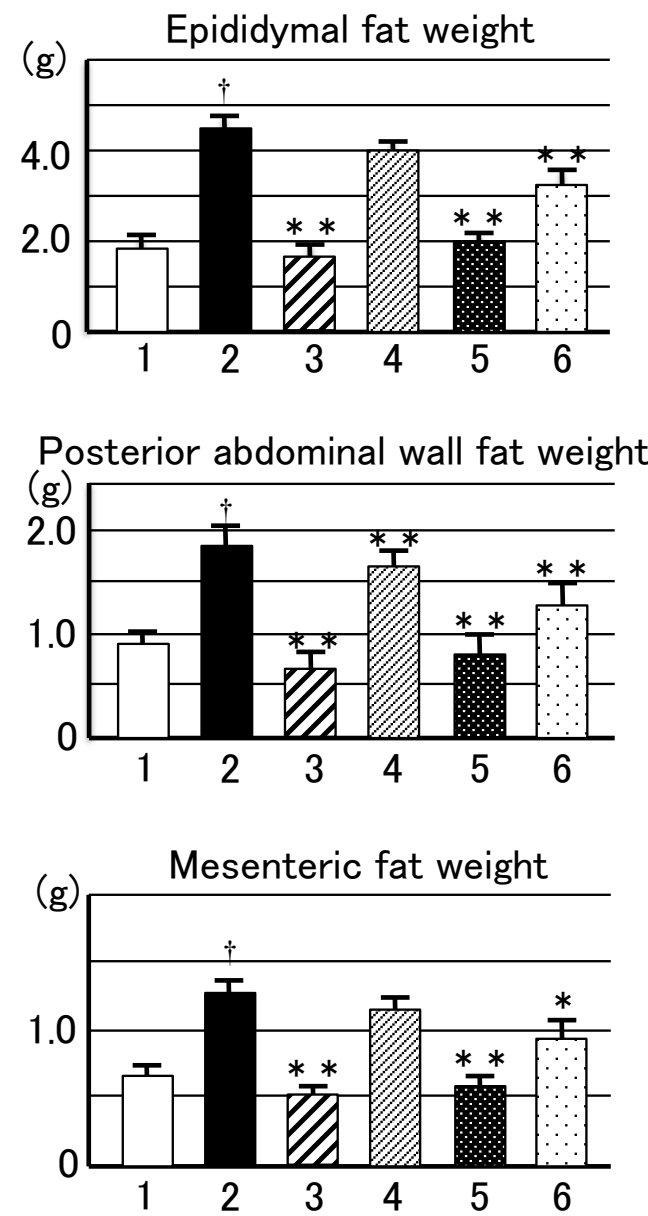

Figure 1. Comparison of Fat Weight (100 g Body Weight) Group of Mice of AOM Administration. Significantly different from Group $1(\dagger \mathrm{p}<0.01$; Student $t$-test); Significantly different from Group $2\left(* * \mathrm{p}<0.01,{ }^{*} \mathrm{p}<0.05\right.$; Dunnett's multiple comparisons test)
Groups 1 and 5. Fat weights (100 g body weight) are shown in Figure 1. They were significantly higher in the epididymis, posterior abdominal wall, and mesentery in Group 2 than in Group $1(\mathrm{p}<0.01)$, and were significantly lower in Groups 3, 5, and 6 than in Group 2 ( $p<0.05$ for the mesentery in Group 6, p <0.01 in the others). In addition, they were significantly lower in the posterior abdominal wall in Group $4(\mathrm{p}<0.01)$.

Liver triglyceride and total cholesterol concentrations

Triglyceride and total cholesterol concentrations in liver tissue are shown in Figure 2. Triglyceride concentrations in liver tissue were significantly higher in Group 2 than in Group 1 ( $\mathrm{p}<0.01)$, and were significantly lower in Groups 3 and 5 than in Group $2(\mathrm{p}<0.01)$. Total cholesterol concentrations were significantly higher in Group 2 than in Group $1(\mathrm{p}<0.01)$, significantly lower in Group 3 than in Group $2(\mathrm{p}<0.05)$, and were decreasing in Group 5.
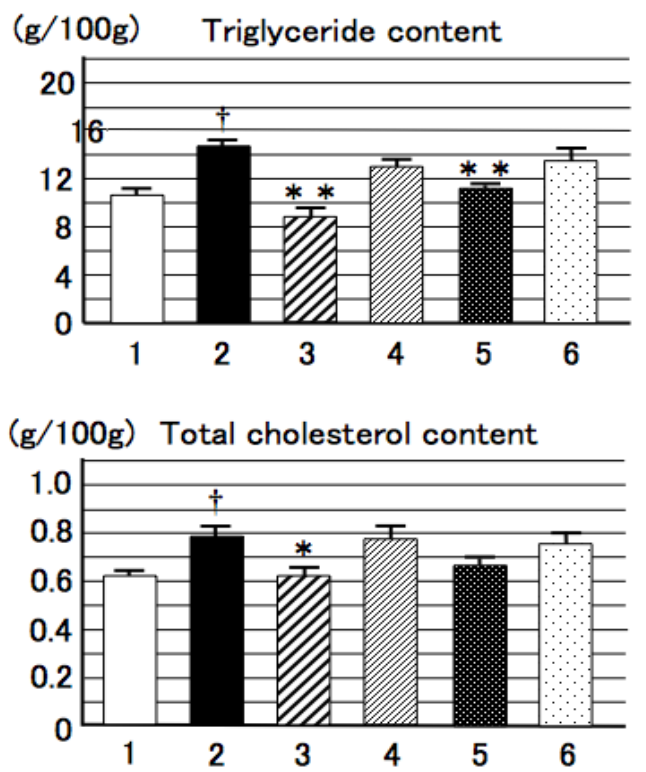

Figure 2. Liver Triglyceride and Total Cholesterol Content. Significantly different from Group $1(\dagger \mathrm{p}<0.01$; Student t-test). Significantly different from Group $2(* * p<0.01$, $* \mathrm{p}<0.05$; Dunnett's multiple comparisons test) 

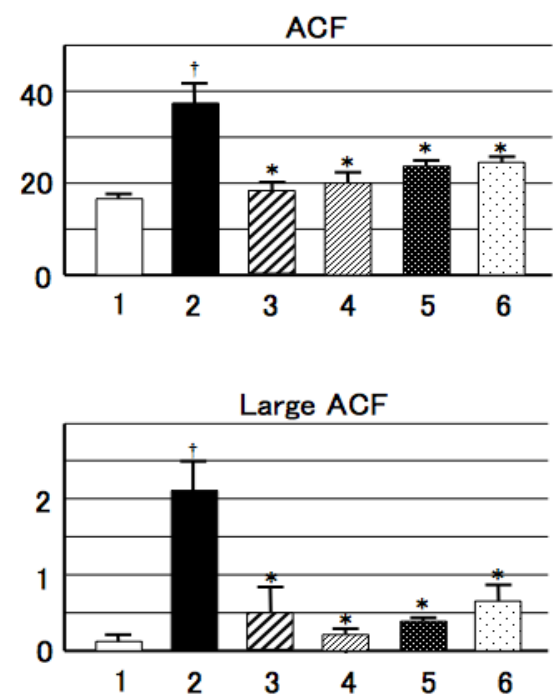

Figure 3. Evaluation of Mouse Colon ACF of AOM Administration Group. Significantly different from Group 1 ( $\dagger \mathrm{p}<0.01$; Student $t$-test); Significantly different from Group $2(* \mathrm{p}<0.01$; Dunnett multiple comparisons test)

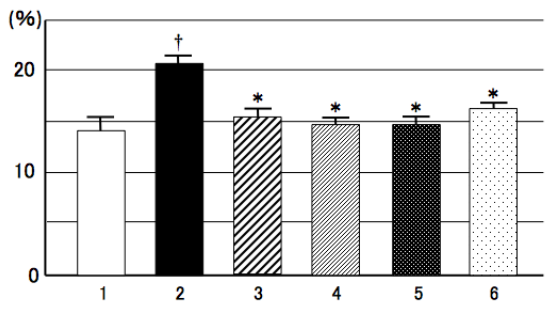

Figure 4. Ki-67 Labeling Index in Normal Colon Mucosa. Significantly different from Group $1(\dagger \mathrm{p}<0.01$; Student $t$-test); Significantly different from Group $2\left({ }^{*} \mathrm{p}<0.01\right.$; Dunnett's multiple comparisons test)

\section{Number of ACF}

The numbers of ACF and large ACF (number of ACF with four or more abnormal crypts per focus) are summarized in Figure 3. All the numbers were significantly higher in Group 2 than in Group $1(\mathrm{p}<0.01)$, and were significantly lower in Groups 3-6 than in Group $2(\mathrm{p}<0.01)$. The ACF inhibition rates were 52, 46, 42, and $40 \%$ in Groups 3, 4,5, and 6, respectively, relative to $100 \%$ in Group 2. IThe numbers of large ACF were decreased by $76,90,86$, and $71 \%$ in Groups 3,4,5, and 6, respectively, relative to $100 \%$ in Group 2.

\section{Ki-67 labeling index}

For assessing cell proliferation in the colon, the Ki67-labeling indices were measured. As shown in Figure 4, the Ki-67-labeling index was significantly higher in Group 1 than in Group $2(\mathrm{p}<0.01)$; inhibition rates were significantly lower, i.e., 26, 30, 31, and $22 \%$ in Groups $3,4,5$, and 6 , respectively, relative to $100 \%$ in Group 2 $(\mathrm{P}<0.01)$.

\section{Discussion}

The effects of Eringi and Bunashimeji on the formation of $\mathrm{ACF}$ (colorectal precancerous lesions) in AOM-induced mice on a high-fat diet were examined. As a result, both 5 and $1 \%$ diets significantly inhibited ACF formation, and its mean inhibition rate was as high as $45 \%$. The increased incidence of colon cancer is caused by excessive calorie intake due to westernization of the diet and obesity due to animal fat intake (Endo et al., 2013). The incidence of colon cancer increases by $7 \%$ when the body mass index (BMI), an indicator of obesity, increases by 2 (Calle et al., 2004). In this experiment, $5 \%$ Eringi and Bunashimeji diets significantly suppressed the increase in body weight due to a high-fat diet at one week after the start of the experiment until the end. Fat weights were significantly suppressed in the epididymis, posterior abdominal wall, and mesentery. They were significantly suppressed in the posterior abdominal wall in the $1 \%$ Eringi group and in all regions in the $1 \%$ Bunashimeji group. Plasma triglyceride and total cholesterol levels were not significantly increased in the high-fat diet group at that time, as compared with the normal diet group, precluding the examination of the effects of Eringi and Bunashimeji (data not shown). Triglyceride levels were not elevated in the high-fat diet group, suggesting that the ACF-inhibiting effects observed in this experiment were not caused by the pancreatic lipase activity-lowering effects of Eringi and Bunashimeji.

Subsequently, the liver, whose absolute weight was significantly lower and relative weight showed no change, was histopathologically examined, demonstrating that lipid droplet formation was suppressed in the 5\% Eringi and Bunashimeji groups (data not shown). Triglyceride and total cholesterol levels in liver tissue were measured, demonstrating that triglyceride levels were significantly lower in the 5\% Eringi group and triglyceride and total cholesterol levels were significantly lower in the 5\% Bunashimeji group. Thus, the antiobesity effects of Eringi and Bunashimeji may be involved in the suppression of ACF formation.

Visceral fat accumulation due to obesity and abnormal adipocytokine secretion from fat cells are involved in colon carcinogenesis (Endo et al., 2013). In the 5\% Eringi and Bunashimeji groups, fat weights were significantly lower in the epididymis, posterior abdominal wall, and mesentery. Also, triglyceride and total cholesterol levels in the liver were significantly lower, demonstrating inhibitory effects on liver fat accumulation. Thus, the suppression of visceral fat accumulation may improve abnormal adipocytokine secretion. Adiponectin, a kind of adipocytokine, is an important inhibitor of cell proliferation (Endo et al., 2013). A significant negative correlation between adiponectin and the numbers of ACF has already been reported (Takahashi et al., 2009; Otake et al., 2005). Adiponectin may have inhibitory effects on early carcinogenesis. Leptin, another adipocytokine, inhibits food intake and increases peripheral energy consumption (Lin et al., 2000; Hung et al., 2004). Leptinemia is associated with increased colon cancer risks. However, this should be examined in animal experiments (Endo et al., 2013). In the present study, the adipocytokine level was not measured. In the future, the effects of Eringi and Bunashimeji on adipocytokines will be examined to investigate its effects on the suppression of ACF formation.

The Ki-67 labeling index, a cell proliferation marker of the colonic mucosa, was significantly suppressed in 
both 1 and 5\% Eringi and Bunashimeji groups, and the inhibition rate was concentration-independent and $27 \%$ on average. Inhibitory effects were comparable between the 1 and $5 \%$ diets, suggesting the potent cytostatic activities of Eringi and Bunashimeji as the primary mechanism of the ACF-inhibiting effects observed in the present study.

In AOM-induced mice on a high-fat diet, ACF (precancerous lesion of the colon) formation was significantly inhibited by the $5 \%$ Eringi or $1 \%$ Bunashimeji diet. In a previous experiment with a high-fat diet, an Eringi extract suppressed pancreatic lipase activity and lowered serum triglyceride levels (Mizutani et al., 2008). However, in the present study, plasma triglyceride levels were not elevated in the high-fat diet groups, suggesting that the inhibitory effects of Eringi and Bunashimeji on liver-fat accumulation and cell proliferation were not be mediated by pancreatic lipase. This will be further investigated in the future, because of potential effects on adipocytokines. Eringi and Bunashimeji may be effective for preventing visceral fat obesity, a risk factor of colon carcinogenesis.

\section{Acknowledgements}

This work was partly supported by a Grant from Hokuto Foundation for Bioscience (2012) to T. K.

\section{References}

Bardou M, Barkun AN, Martel M (2013). Obesity and colorectal cancer. Gut, 62, 933-47.

Calle EE, Kaaks R (2004). Overweight, obesity and cancer: epidemiological evidence and proposed mechanisms. Nat Rev Cancer, 4, 579-91.

Cancer Information Service (2015). Statistical information of cancers in different parts of body. http://ganjoho.jp/public/ statistics/pub/statistics04.html (in Japanese). Accessed 1 March 2015.

Edvardsson U, Ljungberg A, Lindén D, et al (2006). PPAR $\alpha$ activation increases triglyceride mass and adipose differentiation-related protein in hepatocytes. J Lipid Res, 47, 329-40.

Endo H, Higurashi T, Takahashi H, Nakajima A (2013). Obesity, life style-related diseases and colorectal cancer. Gastroenterol Endosc, 55, 3735-44.

Endo H, Hosono K, Fujisawa T, et al (2009). Involvement of JNK pathway in the promotion of the early stage of colorectal carcinogenesis under high-fat dietary conditions. Gut, $\mathbf{5 8}$, 1637-43.

Guillamón E, Garcia-Lafuente A, Lozano M, et al (2010). Edible mushroom: role in the prevention of cardiovascular diseases. Fitoterapia, 81, 715-23.

Huang XF, Xin X, McLennan P, Storlien L (2004). Role of fat amount and type in ameliorating diet-induced obesity: insights at the level of hypothalamic arcuate nucleus leptin receptor, neuropeptide $\mathrm{Y}$ and pro-opiomelanocortin mRNA expression. Diabetes Obes Metab, 6, 35-44.

Ikekawa T, Saitoh H, Feng W, et al (1992). Antitumor activity of Hypsizigus marmoreus. I. Antitumor activity of extracts and polysaccharides. Chem Pharm Bull, 40, 1954-7.

Jayasuriya WJABN, Suresh TS, Thusitha D, et al (2012). Oral hypoglycemic activity of culinary-medicinal mushrooms Pleurotus ostreatus and P. cystidiosus (Higher Basidiomycetes) in normal and alloxan-induced diabetic
Wistar rats. Int J Med Mushrooms, 14, 347-55.

Jeong SC, Jeong YT, Yang BK, et al (2010). White button mushroom (Agaricus bisporus) lowers blood glucose and cholesterol levels in diabetic and hypercholesterolemic rats. Nutr Res, 30, 49-56.

Kaneko T, Shimpo K, Chihara T, et al (2012). Inhibition of ENNG-induced pyloric stomach and small intestinal carcinogenesis in mice by high temperature- and pressuretreated garlic. Asian Pac J Cancer Prev, 13, 1983-8.

Katsuki T, Hirata K, Ishikawa H, et al (2006). Aged garlic extract has chemopreventative effects on 1,2-dimethylhydrazineinduced colon tumors in rats. J Nutr, 136, 847s-51s.

Lin S, Thomas TC, Storlien LH, Huang XF (2000). Development of high fat diet-induced obesity and leptin resistance in C57BL/6J mice. Int J Obes Relat Metab Disord, 24, 639-46.

Matsuzawa T, Saitoh H, Sano M, et al (1998). Studies on antioxidant effect of Hypsizigus marmoreus. II. Effects of Hypsizigus marmoreus for antioxidant activities of tumorbearing mice. Yakugaku Zasshi, 118, 476-81.

Matsuzawa T, Sano M, Tomita I, Saitoh H, Ikekawa T (1997). Studies on antioxidant effect of Hypsizigus marmoreus. I. Effects of Hypsizigus mormoreus for antioxidant activities of mice plasma. Yakugaku Zasshi, 117, 623-8 (in Japanese).

McLellan EA, Bird RP (1988). Aberrant crypts: potential preneoplastic lesions in the murine colon. Cancer Res, $\mathbf{4 8 ,}$ 6187-92.

Miyazaki S (2002). Obesity and risk factors. J Clin Sports Med, 19, 112-8 (in Japanese).

Mizutani T, Inatomi S, Kurashima E, Tsuchida T (2008). Suppressive effect of Pleurotus eryngii extract on postprandial serum triacylglycerol elevation. Jpn Pharmacol Ther, 36, 817-23 (in Japanese).

Mori K, Kobayashi C, Tomita T, Inatomi S, Ikeda M (2008). Antiatherosclerotic effect of the edible mushrooms Pleurotus eryngii (Eringi), Grifola frondosa (Maitake), and Hypsizygus marmoreus (Bunashimeji) in apolipoprotein E-deficient mice. Nutr Res, 28, 335-42.

Ohno N, Furukawa M, Miura NN, et al (2001). Antitumor $\beta$ glucan from the cultured fruit body of Agaricus blazei. Biol Pharm Bull, 24, 820-8.

Ohtsuki M, Umeshita K, Kokean Y, et al (2007). Suppressive effects of Bunashimeji (Hypsizigus marmoreus) on triacylglycerol accumulation in C57BL/6J mice. Nippon Shokuhin Kagaku Kogaku Kaishi, 54, 167-72 (in Japanese).

Otake S, Takeda H, Suzuki Y, et al (2005). Association of visceral fat accumulation and plasma adiponectin with colorectal adenoma: evidence for participation of insulin resistance. Clin Cancer Res, 11, 3642-6.

Ren L, Perera C, Hemar Y (2012). Antitumor activity of mushroom polysaccharides: a review. Food Funct, 11, 1118-30.

Sato M, Tokuji Y, Yoneyama S, et al (2013). Effect of dietary maitake (Grifola frondosa) mushrooms on plasma cholesterol and hepatic gene expression in cholesterol-fed mice. J Oleo Sci, 62, 1049-58.

Takahashi H, Takayama T, Yoneda K, et al (2009). Association of visceral fat accumulation and plasma adiponectin with rectal dysplastic aberrant crypt foci in a clinical population. Cancer Sci, 100, 29-32.

Ministry of Health, Labour and Welfare (2012). Vital statistics Japan. Available: http://www.mhlw.go.jp/english/database/ db-hw/index.html. Accessed 28 July 2014. 